\title{
A spatial discounting test to assess impulsivity in dogs
}

\author{
Karen Brady, Lynn Hewison*, Hannah Wright, Helen Zulch, Nina Cracknell, Daniel Mills \\ School of Life Sciences, University of Lincoln, Brayford Pool, Lincoln, LN6 7TS, United Kingdom
}

\section{A R T I C L E I N F O}

\section{Keywords:}

Impulsivity

Spatial discounting

Temperament

Delayed reward

\begin{abstract}
A B S T R A C T
In domestic dog's trait impulsivity can be measured psychometrically using the Dog Impulsivity Assessment Scale (DIAS) and experimentally using a temporal discounting paradigm which requires substantial training. A Spatial Discounting Task (SDT) was developed as an alternative experimental method to assess impulsivity, and evaluated performance in adult (2-10 years) and younger (2-9 months) dogs. The test was modified for field use with fewer controls (Simplified Spatial Discounting Task (SDTs)). Convergent validity with the SDT and DIAS Overall Questionnaire Scores (OQS) and stability over time (4-6 weeks) in the two age groups was determined. $96 \%$ of dogs recruited reached criterion for testing. A significant positive relationship was found between Maximum Distance Travelled (MDT) in the SDT and OQS in adult dogs $(r=0.46, p=0.028)$, with good testretest reliability evident for both $(\mathrm{p}<0.001)$. In young dogs, there was good test-retest reliability for OQS ( $\mathrm{p}=0.023$ ), but no significant relationship was found between OQS and MDT, test-retest reliability for MDT in young dogs was poor. In the SDTs, $100 \%$ of dogs recruited met criterion for testing and there was a significant relationship between MDT and OQS ( $r=0.61, \mathrm{p}=0.027)$. The SDT appears to be a useful method for measuring impulsivity in adult dogs with wide applicability.
\end{abstract}

\section{Introduction}

Impulsivity has been defined as the extent to which an individual evaluates the consequences of their behaviour (Moeller et al., 2001; Barratt and Patton, 1983; Fairbanks et al., 2004; Peremans et al., 2002), and is expressed in a range of behaviours linked with inhibitory control (Logue et al., 1988; De Wit, 2008; Wright et al., 2012). Higher levels of impulsivity have been linked to poorer or less accurate choices in terms of decision making (Dalley et al., 2008; Puumala and Sirvo, 1999) suggesting that this aspect of temperament is potentially an important predictor of performance in complex environments. Temporal discounting (the ability to tolerate delays in reward delivery) has been used to examine temperament (Ostaszewski, 1996), and has extensively been used as a measure of impulsivity across a range of species including rats (Perry et al., 2007; Reynolds et al., 2002; Green et al., 2004) pigeons (Green et al., 2004; Ainslie, 1974; Logue et al., 1988; Wolff and Leander, 2002), dogs (Wright et al., 2012), primates (Tobin et al., 1996; Evans and Beran, 2007; Rosati et al., 2007) and humans (Pine et al., 2010; Dittmar and Bond, 2010; Ostaszewski, 1996; Bickel et al., 2007). Temporal discounting paradigms have been used to examine cognitive processes linked to decision making in terms of goal directed behaviour, addiction behaviours (Bickel et al., 2007), and socially important behaviours (Critchfield and Kollins, 2001).

In addition, impulsivity as a trait is thought to be linked to behaviour problems and disorders relating to a limited or lack of selfcontrol and an inability to tolerate frustration (Brady et al., 1998; Vollmer et al., 1999; Fairbanks et al., 2004; Perry et al., 2005; Pine et al., 2010; Wright et al., 2011; Pavlov et al., 2012). The use of temporal delay discounting to understand cognitive processes enables measurement of traits relating to decision making processes such as impulsivity, but to maximise the potential to do this the tests used to assess these traits need to be practical in a variety of contexts in which they would be useful, such as in the working dog selection environment, which is very different to the laboratory setting, with pressure on both the time and space available for assessment.

The Dog Impulsivity Assessment Scale (DIAS) is a validated questionnaire which assesses impulsivity in dogs in terms of their overall level of the trait (Overall Questionnaire Score, OQS), but also in terms of 3 component factors: Behavioural Regulation (Factor 1), Aggression and Response to Novelty (Factor 2), and Responsiveness (Factor 3) (Wright et al., 2011). While this tool is useful for measuring impulsivity in dogs, it is limited to those with a known history (where there is a consistent caregiver able to answer the questions for the dog over a range of contexts). As part of the validation of the DIAS it has shown convergent validity with an operant learning task centred around a temporal discounting paradigm (delayed reward choice task (DRCT) (Wright et al., 2012)). This examines maximum time delay tolerated for a larger reward when an immediate smaller reward is also available,

\footnotetext{
* Corresponding author.

E-mail address: LHewison@lincoln.ac.uk (L. Hewison).
} 
(Wolff and Leander, 2002). The maximum delay tolerated before switching correlated with the psychometric assessment of impulsivity (more impulsive individuals tolerated shorter delay) (Wright et al., 2012). The long-term stability of impulsivity has also been examined and found to be stable up to 6 years post initial assessment using the DIAS (Reimer et al., 2014).

Unlike the DIAS, the DRCT can be used to generate an impulsivity score in dogs of unknown history, but the test involves considerable training, with dogs reported to take up to four, $60 \mathrm{~min}$ sessions to reach the criterion required before assessment can begin. A 50\% drop out rate has been reported also during this process (Wright et al., 2012). These factors limit the feasibility of this test to practically assess impulsivity (Wilsson and Sundgren, 1998) with the potential for selection bias. Spatial discounting potentially offers a simpler alternative to temporal discounting for the assessment of impulsivity. It assesses the animal's perception of reward value as distance changes (Stevens et al., 2005). The spatial discounting paradigm typically involves assessing the value of reward while gradually increasing the distance an animal is required to travel for a larger reward compared to a smaller closer one. The distance at which the subject will no longer travel for the larger reward and switches to the small, closer reward is used as the metric of interest. Spatial discounting paradigms are not entirely independent of temporal choice, as an increase in distance also creates an increase in time taken to reach the larger reward. Similar to temporal discounting, spatial discounting has been used in a variety of ways to help understand decision making behaviour. It has been used in primates to help examine goal directed behaviour relating to foraging patterns (Kralik and Sampson, 2012), evaluative behaviours in rats (Papale et al., 2012), and the differing impact of food and social reward on decision making behaviour in guppies (Muhlhoff et al., 2011). However, there is also evidence to suggest that spatial and temporal discounting may not completely correlate. In primates, differences have been observed between species (Tamarins and Marmosets) in terms of their delay tolerance in spatial and temporal discounting tasks. While Tamarins travelled further for a large reward compared to Marmosets, the marmosets waited longer for the large reward, this has been suggested to be a result of different feeding ecology between the species (Stevens et al., 2005). These differences between the rates of spatial and temporal discounting in these species suggest there is a difference in the way these types of delay are viewed, and that this varies between species. In relation to the dog, a more opportunist scavenger or hunter, there is no information on the convergence or divergence between spatial and temporal discounting. While temporal discounting has been used to measure temperament traits (Wright et al., 2012), little is known about how spatial delay is tolerated by individuals and whether it can be used to assess temperament traits in a similar way.

Temperament traits arise largely as a result of the interaction of genetic and experiential factors early in life, it has been shown to change over time from early life in both humans (Putnam et al., 2001) and primates (Heath-Lang et al., 1999), but it is still largely unknown at what age many traits become stable and predictable in dogs. In dogs, it is unknown at what age the DIAS or the delayed reward choice task are valid, but it is important to understand this, as selection for future work function often occurs early in the dog's life (Svobodová et al., 2008; Wilsson and Sundgren, 1998). The advantages of a spatial discounting task over a temporal delay paradigm are that no specific training is necessarily required, giving the resulting test the potential to be applied more quickly, with a greater number of animals able to pass inclusion criteria for assessment. This could make it a simpler tool for use in younger animals and those with less training history.

Therefore, the aim of this study was to develop a spatial discounting task suitable for dogs of all ages and to validate this against DIAS scores in adult dogs. Secondarily, we examined the validity of the spatial discounting task and DIAS as a means of assessing impulsivity in young dogs. Finally, we adapted the spatial discounting task to make it simpler to use in a field setting with fewer controls and examined its convergent validity with DIAS.

Two variants of a runway task were used to examine the distance at which a large reward was discounted by dogs, in favour of a smaller closer reward. This measure from each variant was then examined against overall questionnaire score (OQS) for impulsivity to establish the preferred form of runway method for assessing impulsivity in dogs and puppies. DIAS scores and spatial discounting task performance in dogs under 9 months of age were then examined to investigate the development of the trait over time within individuals.

\section{Material and methods}

Informed consent was obtained from dog owners prior to the study. The University of Lincoln ethical procedures and guidelines were adhered to and approval was obtained before data collection.

\subsection{General principles of the spatial discounting task (SDT) used}

In the spatial discounting task (SDT), a variant of the spatial discounting paradigm, dogs are presented with two trays, one containing 3 pieces of food, and the other containing 1 piece of food. Training involves ensuring the dog can discriminate between where the large and small rewards are located. Once this has been established, testing involves a series of trials in which the dog is allowed to choose between the two trays. The dog naturally chooses the tray with the larger reward initially, but this tray is gradually moved further away from the dog. The goal is to establish the maximum distance the dog will travel for the larger reward before switching to the smaller, closer reward, defined by a predetermined number of consecutive choices of this smaller reward (Figs. 1 and 2).

In our version, the trays were of different colour and luminance to ease discrimination and the larger reward tray moved $25 \mathrm{~cm}$ at a time. Further details of the specific methods and how they were adapted is given in relation to each study below.

\subsubsection{Psychometric profiling}

Owners completed The Dog Impulsivity Assessment Scale (DIAS) (Wright et al., 2011) to enable impulsivity scores and related components to be compared against the maximum distance they travelled. For dogs which completed the spatial discounting task on two separate occasions, the questionnaire was also completed twice to examine the test retest reliability of the DIAS in dogs under 9 months of age. From the DIAS, Overall Questionnaire Score (OQS), Factor 1 Behavioural Regulation (F1), Factor 2 Aggression and Response to Novelty (F2), and Factor 3 Responsiveness (F3) were all calculated as measures of components of impulsivity as per Wright et al. (2011).

\subsection{Laboratory study 1: spatial discounting task (SDT1) with adult dogs}

\subsubsection{Subjects}

25 adult dogs over 2 years old were recruited from the general public, 24 of which reached criterion for data inclusion. Half of the subjects (12 adult dogs) were retested 4-6 weeks after initial testing.

\subsubsection{Equipment}

Two equal sized trays (length $36 \mathrm{~cm} \times$ width $25 \mathrm{~cm} \times$ depth $6 \mathrm{~cm}$ ), one blue, one yellow, were used, with the larger reward consisting of 3 pieces of cheese, and the smaller one 1 piece of cheese (approx. $1 \mathrm{~cm}^{3}$ ). The colour of the tray containing each reward was randomised between subjects within the constraints of counterbalancing across the population for both colour of tray and contents.

A tape measure was attached to the floor to a length of $10 \mathrm{~m}$ to enable the experimenter to rapidly set the trays up at the start, move the large reward tray accurately during testing, and measure the Maximum Distance Travelled (MDT) by the dog. Each tray was positioned lengthwise, one $25 \mathrm{~cm}$ to the left, with the front of the tray 


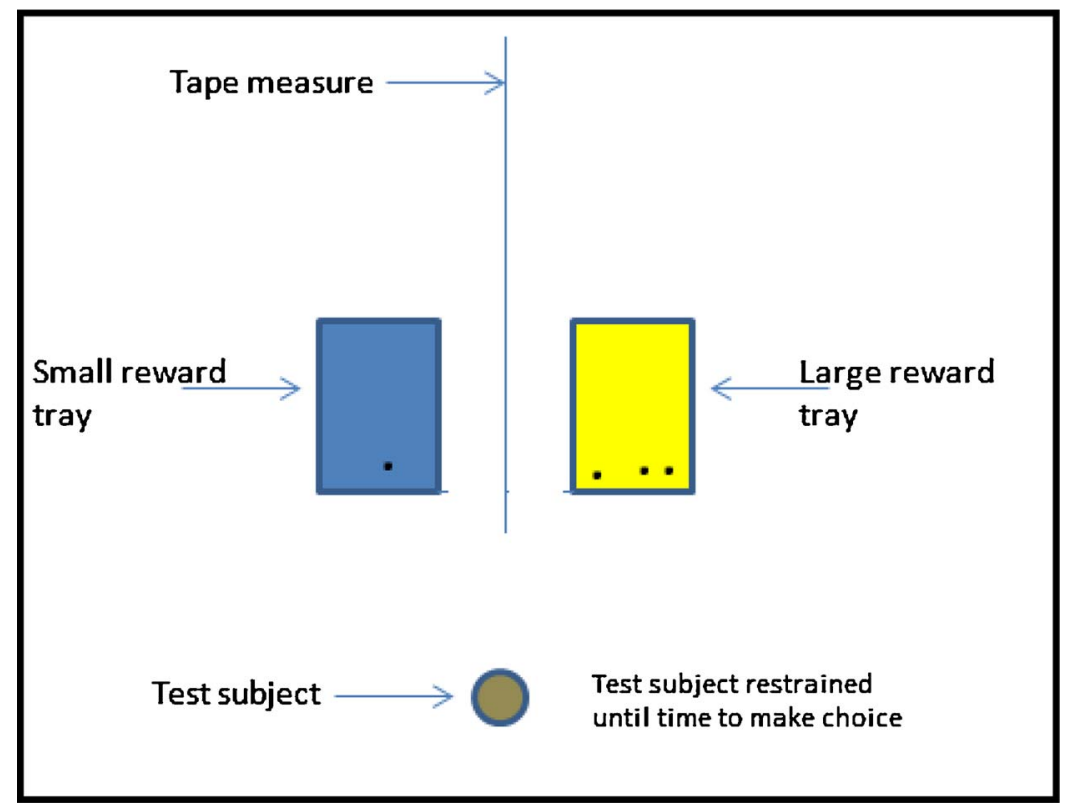

Fig. 1. Experimental set up for the spatial discounting task (SDT).

parallel to the start of the tape, the other tray positioned $25 \mathrm{~cm}$ to the right in the same way. An opaque screen $(50 \mathrm{~cm}$ high) running the length of the tape was used to ensure that once the dog had made a choice it could not change that decision and switch sides. Test subjects were positioned $1 \mathrm{~m}$ in front of the start position of the trays.

A curtain ( $80 \mathrm{~cm}$ high) was used to block each dog's visual access to the equipment when not being tested. This was positioned between the test subject and the start of the tape measure and trays. Before each trial the curtain was dropped to allow the dog to approach the test set up and after each trial the dog was taken back behind the start line and the curtain was put back up.

Dogs were restrained on a short lead that was attached to a secured, static point behind the experimental set up when not making a choice. When required to perform the task they were swapped from the short lead onto a long line and the curtain was dropped to allow the dog to approach the experimental set up and make a choice. The line was held by the researcher from behind the dog. Once a tray had been chosen, lead length was reduced and the dog was then taken back behind the start line and re-secured. The curtain was then put back up and the rewards replenished and equipment repositioned (if the large reward tray had been selected). The researcher operated the curtain and managed the dog while an assistant replenished rewards and repositioned the equipment. Training and testing stages were conducted within a controlled enclosed space at the University of Lincoln Animal Behaviour and Training Centre.

\subsubsection{Training protocol for spatial discounting task (SDT)}

Both trays were presented simultaneously for 12 trials (dual-presentation trials). Testing only occurred if the dog met criterion by choosing the tray containing the larger reward at least 10/12 times in 2 successive sets of dual-presentation trials, the last 5 choices in each trial also had to be for the larger reward tray. Dogs were given up to 5 sets of dual presentation trials to reach criteria.

\subsubsection{Testing: distance choice test}

Testing began following a 10-min break after meeting criterion.

This stage involved repeated presentation of both trays (Fig. 2). Each time the large reward tray was chosen it was moved further from

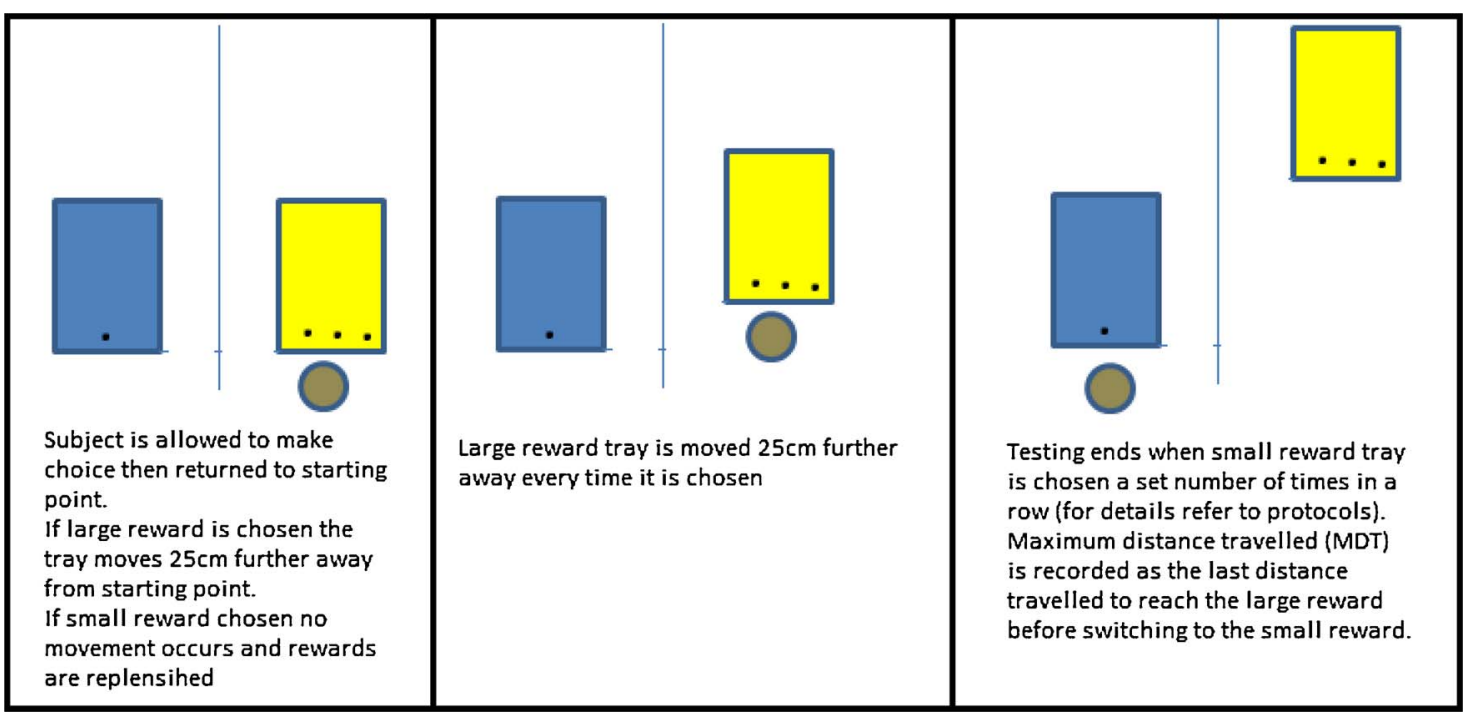

Fig. 2. Testing procedure for the spatial discounting task (SDT). 
the start line by $25 \mathrm{~cm}$. When the small reward tray was chosen, the position of the trays remained the same for the next trial; rewards were then simply replenished. This testing stage ended when the dog had chosen the small reward tray 5 times in succession. At this point, the distance to the large reward tray was measured and recorded as the MDT. All MDT measurements were taken from the front of the tray.

All dogs were required to choose the tray containing the large reward at least once during test trials for their MDT results to be considered valid.

\subsection{Simplified field study: SDTs with adult dogs}

\subsubsection{Subjects}

13 dogs aged between 2 years and 9 years were recruited from the general public to take part in a simplified version of the SDT, referred to as a Simplified Spatial Discounting Task (SDTs). This involved the following changes:

Training and testing was carried out in an environment familiar to each dog, usually in the garden or driveway of their home, rather than in a controlled setting. There was no barrier nor curtain. Dogs were trained to discriminate between a blue, large reward tray ( 3 pieces of preferred food of the subject, rather than cheese) and yellow, small reward tray (1 piece of the same preferred food of the subject) by presenting the large reward tray thirty times followed by the small reward tray ten times. Training criteria was met when the dog chose the large reward tray at least $8 / 10$ times in 2 successive dual presentation trials. The dog then progressed to the testing phase (as described in 2.2.4) which ended when the dog had chosen the small reward tray 10 times in succession.

\subsection{Laboratory study 2: SDT2 with young dogs}

\subsubsection{Subjects}

25 dogs aged between 2 and 9 months were recruited from the general public, 24 of which reached criterion for data inclusion. Half of the subjects (12) were retested 4-6 weeks after initial testing.

The SDT protocol from Laboratory Study 1 was followed, except that the pieces of cheese were $0.5 \mathrm{~cm}^{3}$, i.e. $1 / 8$ th of the volume.

\subsubsection{Data \& statistical analysis (SPSS 21.0)}

Linear regression was performed on the data to assess relationships between DIAS OQS and MDT in all sample populations, and also to assess relationships between the test and retest for the OQS and MDT in the laboratory studies. Pearson's correlations were also used to determine the strength of the correlation between MDT and OQS in all sample populations.

\section{Results}

\subsection{Laboratory study 1: SDT1 with adult dogs}

Of the 25 dogs recruited, 24 (96\%) reached criterion and completed the SDTl. 1 adult dog was excluded from analysis due to being diagnosed with hip dysplasia shortly after the testing. Accordingly, 23 out of the 24 dogs provided data for analysis of convergent validity with DIAS. The demographic details of age, breed, sex, OQS and MDT for these 24 dogs is shown in Fig. 3. Eleven out of 12 dogs provided data for testretest analysis. The dog that was excluded from both sets of analysis completed the test, but was discovered to have an orthopaedic problem that could have impacted on the results.

The mean MDT for adult dogs was $3.27 \mathrm{~m} \pm 1.74 \mathrm{~m}$ and the mean OQS for adult dogs was $0.55 \pm 0.1$.

A significant negative correlation $(r=-0.46, p=0.028$, adj $\mathrm{R}^{2}=0.171$ ) was found between OQS and MDT (Fig. 4).

\begin{tabular}{|c|c|c|c|c|}
\hline Age (months) & Sex & Breed & MDT (M) & OQS \\
\hline 24 & $M$ & Labrador & 0.25 & 0.74 \\
\hline 36 & $M$ & Crossbreed & 1.5 & 0.53 \\
\hline 96 & $M$ & Springer Spaniel & 1.75 & 0.39 \\
\hline 48 & $\mathrm{~F}$ & Mini Schnauzer & 1.75 & 0.67 \\
\hline 36 & $\mathrm{~F}$ & Sheltie & 2 & 0.74 \\
\hline 42 & $M$ & Crossbreed & 2 & 0.42 \\
\hline 76 & $\mathrm{~F}$ & Border Collie & 2.25 & 0.34 \\
\hline 28 & $M$ & Border Collie & 2.5 & 0.52 \\
\hline 96 & $\mathrm{~F}$ & Labrador & 2.75 & 0.49 \\
\hline 61 & $\mathrm{~F}$ & Crossbreed & 3 & 0.6 \\
\hline 48 & $\mathrm{~F}$ & Border Collie & 3 & 0.65 \\
\hline 86 & $M$ & Rottweiler & 3.25 & 0.63 \\
\hline 42 & $\mathrm{~F}$ & Sheltie & 3.5 & 0.5 \\
\hline 50 & $M$ & Jack Russell Terrier & 3.5 & 0.49 \\
\hline 52 & $M$ & Golden Retriever & 3.75 & 0.59 \\
\hline 102 & $M$ & Labrador & 4 & 0.47 \\
\hline 26 & $\mathrm{~F}$ & Springer Spaniel & 4.5 & 0.53 \\
\hline 38 & $\mathrm{~F}$ & Jack Russell Terrier & 4.5 & 0.49 \\
\hline 41 & $M$ & Crossbreed & 3.5 & 0.64 \\
\hline 118 & $\mathrm{~F}$ & Crossbreed & 5.75 & 0.41 \\
\hline 108 & $M$ & Springer Spaniel & 6.75 & 0.46 \\
\hline 62 & $M$ & Labrador & 7.5 & 0.45 \\
\hline 71 & $M$ & Crossbreed & 3.25 & 0.61 \\
\hline 84 & $M$ & Crossbreed & 1 & 0.54 \\
\hline
\end{tabular}

Fig. 3. Demographics (age, sex, breed) and Overall Questionairre Score (OQS) from the Dog Impulsivity Assessment Scale (DIAS) and Maximum Distance Travelled (MDT) of Adult Dogs in Laboratory Study 1: SDT1.

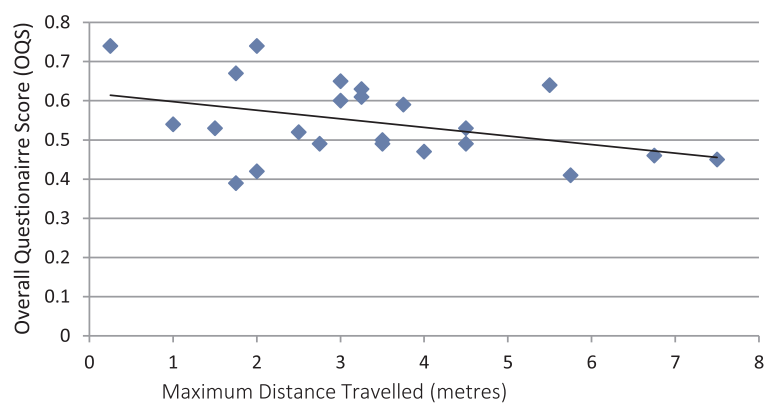

Fig. 4. Relationship between Overall Questionairre Score (OQS) from the Dog Impulsivity Assessment Scale (DIAS) and Maximum Distance Travelled (MDT) in the Spatial Discounting Task (SDT) by adult dogs.

\subsubsection{Test retest}

The relationship between OQS at test and retest in adult dogs was significant $\left(\mathrm{r}=0.949, \mathrm{p}<0.0001\right.$, adj. $\left.\mathrm{R}^{2}=0.89\right)$. The mean OQS at initial testing was 0.54 and the mean OQS at retest was 0.53 .

The relationship between MDT at test and retest in adult dogs was also significant $\left(r=0.859, \mathrm{p}=0.001\right.$, adj. $\left.\mathrm{R}^{2}=0.71\right)$. The mean MDT at initial testing was $3.27 \mathrm{~m}$ and the mean MDT at retest was $3.55 \mathrm{~m}$ (Fig. 5).

\subsubsection{DIAS factor analysis}

A significant relationship was also found between MDT and DIAS Factor $1\left(\mathrm{r}=-0.463, \mathrm{p}=0.026\right.$, adj. $\mathrm{R}^{2}=0.177 \mathrm{Fig}$. 6).

No Significant relationships were found between MDT and DIAS Factor $2(\mathrm{p}=0.971)$ or MDT and Factor $3(\mathrm{p}=0.296)$.

\subsubsection{Simplified field study: SDTs}

All 13 dogs recruited met criteria for testing and all provided data for analysis. The demographic details of age, breed, sex, OQS and MDT for these 13 dogs is shown in Fig. 7.

The mean MDT was $1.88 \mathrm{~m} \pm 1.64 \mathrm{~m}$ and the mean OQS was $0.53 \pm 0.07$. A significant negative correlation $(r=-0.61, p=0.028$, 


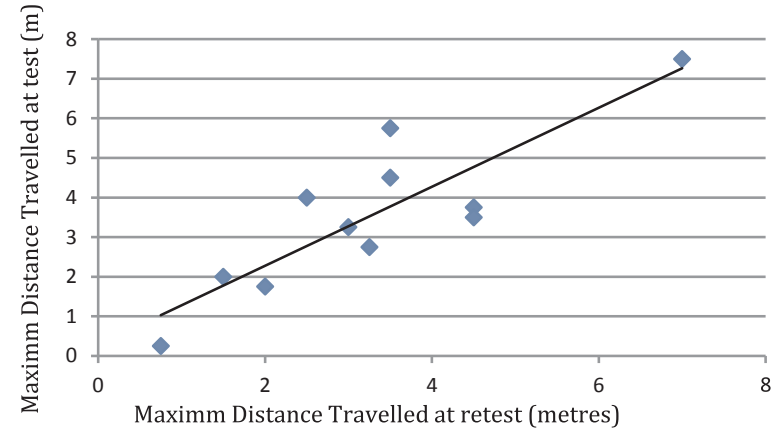

Fig. 5. Relationship between Maximm Distance Travelled (MDT) from the Spatial Discounting Task (SDT) at test and retest by adult dogs.

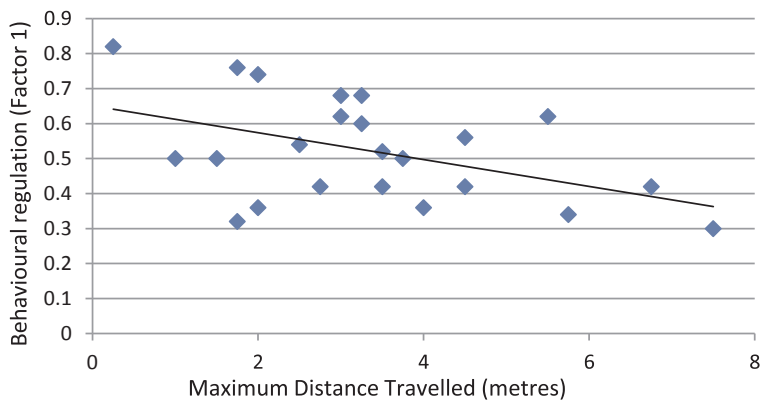

Fig. 6. Relationship between Maximum Distance Travelled (MDT) from the Spatial Discounting Task (SDT) and Behavioural Regulation (Factor 1 from the Dog Impulsivity Assessment Scale (DIAS)) by adult dogs.

\begin{tabular}{|r|l|l|r|r|}
\hline Age (months) & Sex & Breed & MDT (M) & OQS \\
\hline 108 & M & West Highland White Terrier & 1 & 0.56 \\
\hline 108 & M & Cross breed & 0.75 & 0.62 \\
\hline 84 & M & Cross breed & 5 & 0.43 \\
\hline 48 & F & German Shepherd & 1.5 & 0.59 \\
\hline 36 & F & Cross breed & 1.5 & 0.49 \\
\hline 48 & F & German Shepherd & 0.75 & 0.64 \\
\hline 72 & M & Border Collie & 0.75 & 0.49 \\
\hline 84 & M & Border Collie & 1 & 0.62 \\
\hline 60 & F & Jack Russel Terrier & 0.25 & 0.49 \\
\hline 72 & F & Jack Russel Terrier & 3.75 & 0.50 \\
\hline 96 & M & Australian Shepherd & 3.25 & 0.50 \\
\hline 36 & M & Labrador & 0.5 & 0.58 \\
\hline 48 & M & Yorkshire Terrier & 4.5 & 0.43 \\
\hline
\end{tabular}

Fig. 7. Demographics (age, sex, breed) and Overall Questionairre Score (OQS) from the Dog Impulsivity Assessment Scale (DIAS) and Maximum Distance Travelled (MDT) of Adult Dogs in the Simplified Field Study: SDT.

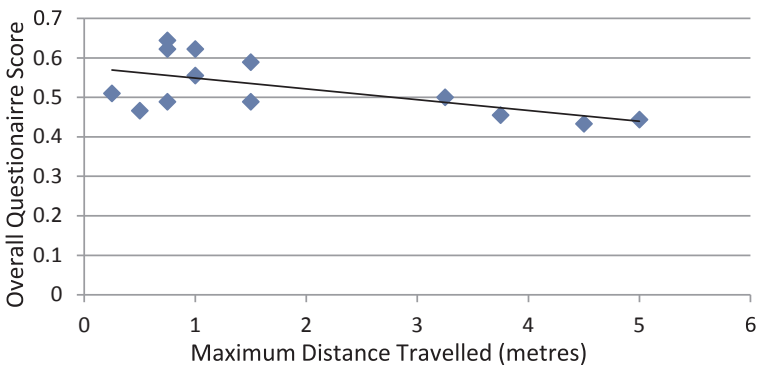

Fig. 8. Relationship between Overall Questionairre Score (OQS) from the Dog Impuslviity Assessment Scale (DIAS) and Maximum Distance Travelled (MDT) from the Simplified Spatial Discounting Task (SDTs) by adult dogs.

adj. $\mathrm{R}^{2}=0.313$ ) was found between OQS and MDT (Fig. 8).

No Significant relationships were found between MDT and Factor 1 $(p=0.162)$, Factor $2(p=0.192)$ or Factor $3(p=0.154)$.

\begin{tabular}{|r|l|l|r|r|}
\hline Age (months) & Sex & Breed & MDT (M) & OQS \\
\hline 3 & F & German Shepherd & 2 & 0.59 \\
\hline 2 & F & Labrador & 1.25 & 0.56 \\
\hline 2 & F & Golden Retriever & 0.25 & 0.59 \\
\hline 3 & F & Cavapoo & 2.5 & 0.56 \\
\hline 5 & F & Labrador & 5.25 & 0.52 \\
\hline 4 & M & Mini Schnauzer & 2.75 & 0.47 \\
\hline 4 & M & Labrador & 7 & 0.61 \\
\hline 3 & F & Border Terrier & 1.7 & 0.66 \\
\hline 4 & M & Springador & 3 & 0.44 \\
\hline 3 & M & Shih Tzu & 0 & 0.58 \\
\hline 4 & F & Crested Powder Puff & 2 & 0.46 \\
\hline 3 & F & American Bulldog & 2.75 & 0.69 \\
\hline 2 & M & Springer Spaniel & 1.5 & 0.46 \\
\hline 3 & F & Crossbreed & 3.25 & 0.54 \\
\hline 3 & F & Lurcher & 5.5 & 0.53 \\
\hline 3 & M & Cockapoo & 3.25 & 0.59 \\
\hline 3 & M & Springer Spaniel & 4.75 & 0.57 \\
\hline 2 & M & Large Munsterlander & 0 & 0.53 \\
\hline 4 & F & Mallinois & 0.25 & 0.59 \\
\hline 4 & F & Greyhound & 1.25 & 0.46 \\
\hline 4 & M & Crossbreed & 0.55 \\
\hline 3 & M & Cockapoo & 0.47 \\
\hline 4 & M & Border Collie & 0.52 \\
\hline & & & \\
\hline
\end{tabular}

Fig. 9. Demographics (age, sex, breed) and Overall Questionairre Score (OQS) from the Dog Impulsivity Assessment Scale (DIAS) and Maximum Distance Travelled (MDT) of Young Dogs in Laboratory Study 2: SDT2.

\subsection{Laboratory study 2: SDT2 with young dogs}

Of the 25 young dogs recruited, 24 (96\%) reached criterion and completed the behaviour test (SDT). Twenty three out of the 24 young dog data were used for analysis, 11/12 retest data were analysed. One of the young dogs was excluded from analysis after having completed the testing as they had come into season during the retest and this may have impacted on their results. The demographic details of age, breed, sex, OQS and MDT for the 23 young dogs is shown in Fig. 9.

The mean MDT in young dogs was $2.70 \mathrm{~m} \pm 1.94 \mathrm{~m}$ and the mean OQS was $0.54 \pm 0.06$. The relationship between OQS and MDT for puppies was not significant $\left(r=-0.053, p=0.811\right.$, adj. $R^{2}=-0.062$ Fig. 10).

\subsubsection{Test retest}

The relationship between OQS at test and retest in young dogs was significant $\left(r=0.673, p=0.023\right.$, adj. $\left.R^{2}=0.393\right)$. The mean OQS at test was 0.54 and the mean OQS at retest was 0.57. See Fig. 11 .

The relationship between MDT at test and retest in puppies was not significant $\left(\mathrm{r}=0.414, \mathrm{p}=0.15\right.$, adj. $\left.\mathrm{R}^{2}=0.115\right)$. The mean MDT at test was $3.09 \mathrm{~m}$ and the mean MDT at retest was $2.80 \mathrm{~m}$ (Fig. 12).

A significant positive correlation $(r=0.62, p=0.044$, adj. $\mathrm{R}^{2}=310$ ) was found between DIAS Factor 3 and MDT in young dogs in

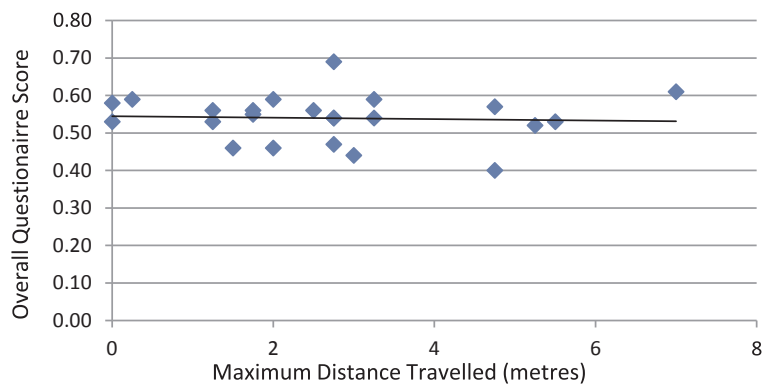

Fig. 10. Relationship between Overall Questionairre Score (OQS) from the Dog Impulsivity Assessment Scale (DIAS) and Maximum Distance Travelled (MDT) in the Spatial Discounting Task (SDT) by young dogs. 


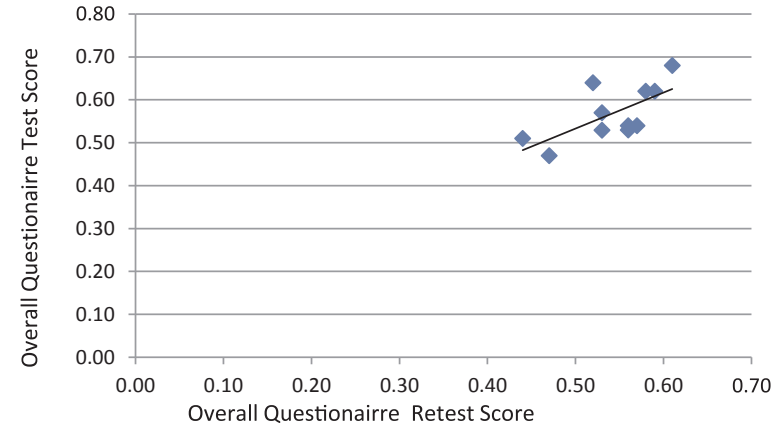

Fig. 11. Relationship between Overall Questionairre Score (OQS) from the Dog Impulsivity Assessment Scale (DIAS) at test and retest by young dogs.

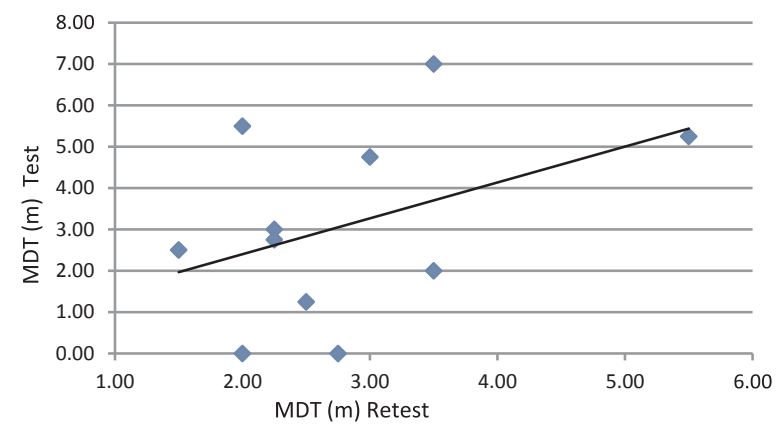

Fig. 12. Relationship between Maximum Distance Travelled (MDT) in the Spatial Discounting Task (SDT) at test and retest by young dogs.

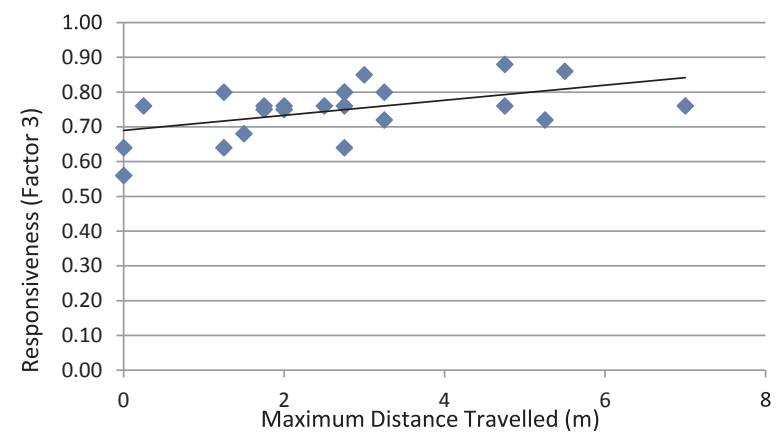

Fig. 13. Relationship between Maximum Distance Travelled (MDT) in the Spatial Discounting Task (SDT) and Behavioural Responsiveness (Factor 3 from the Dog Impulsivity Assessment Scale (DIAS)) by young dogs.

the retest (Fig. 13), but not for the initial test $(r=0.421, p=0.197$, adj. $\mathrm{R}^{2}=0.086$.

No significant relationships were found between MDT and DIAS Factor $1(\mathrm{p}=0.59)$ or Factor $2(\mathrm{p}=0.84)$.

\section{Discussion}

This study successfully developed a spatial discounting task suitable for use in dogs, and has shown that it has reliability and convergent validity with DIAS in adult dogs. However, there are still challenges relating to the assessment of impulsivity in young dogs. While the DIAS was found to be a reliable tool, the age at which the spatial discounting task can be used to measure the trait in dogs remains unknown.

Convergent validity was found between the behaviour test and OQS $(\mathrm{p}=0.028)$ and DIAS Factor 1 (Behavioural Regulation) $(\mathrm{p}=0.026)$ in the controlled laboratory study (SDT1). In practical terms this meant that dogs that travelled a shorter distance before switching (and thus tolerated a shorter delay for reward) had higher impulsivity scores compared to those that travelled further in the behaviour test; this effect has previously been demonstrated in a temporal discounting task in dogs (Wright et al., 2012). The spatial discounting task also showed reliability with MDT measures used in the controlled laboratory test after 4-6 weeks among adult dogs. In light of this the behaviour test could be used to predict impulsivity as measured by OQS in adult dogs when it is not suitable for the DIAS to be completed, for example when a dog does not have a consistent caregiver to answer the questionnaire. Spatial discounting can be used to assess impulsivity in dogs and yields similar results to previous research which used a temporal discounting paradigm (Wright et al., 2012), suggesting a degree of convergence between spatial and temporal discounting in dogs, unlike some species of primate (Stevens et al., 2005).

Relationships between temporal discounting and temperament traits relating to impulsivity have been observed in a variety of species (Ostaszewski, 1996; Perry et al., 2007; Ainslie, 1974; Wolff and Leander, 2002; Tobin et al., 1996; Evans and Beran, 2007; Rosati et al., 2007; Bickel et al., 2007). Our research shows individual differences in tolerance to spatial delay of reward in dogs, linked to how impulsive the individual is. The convergence between spatial and temporal delay tolerance in dogs, rather than the primate species studied, could potentially be linked to their feeding ecology as hunters or scavengers, which may have created an adaptive advantage to being flexible in terms of the nature of delays they can tolerate. The individual differences in the rate of discounting are potentially linked to differences in time perception or susceptibility to rewards, which impact on decision making processes. This could help with the way we select and train dogs for working roles, and enable us to better predict and manage problem behaviours relating to impulse control.

Both laboratory and field versions of the spatial discounting task produced similar results in terms of the relationship between MDT and OQS. As fewer controls were used in the field study this suggests that the test is quite robust in the face of environmental changes, and that the distance and reward are most important in controlling the outcome rather than other environmental factors or distractors. The spatial discounting task is a simple test that can be used to assess impulsivity in adult dogs, it is a faster and easier method for assessing impulsivity in dogs compared to previous laboratory assessments using temporal discounting. The maximum time for completion of the task was one $2 \mathrm{~h}$ session and on average $96 \%$ of dogs recruited across the 3 studies were able to complete testing. Being quick and simple for the dogs to engage with improves the way the test can be utilised. Results of the field adaptation also show that a simplified version of the spatial discounting task can be a valid way of assessing impulsivity in a less controlled setting without the need for so many controls. No relationship was found between the field behaviour test and Factor 1 (Behavioural Regulation), while this could be a result of the smaller sample size, it could also indicate that the field test cannot provide the same level of detail relating to assessment of the trait compared to the laboratory test. However, with a higher proportion of dogs meeting criteria for testing, and a short, single training session required to complete the test, the spatial discounting test is a more time feasible method of measuring impulsivity in domestic dogs, compared to the delayed reward choice task (Wright et al., 2012).

In humans, reward value has been shown to influence the temporal delay tolerated (Rosati et al., 2007). However, reward ratio has been shown to be more important than absolute values in primates, with 1 treat used as a small reward and 3 as the large reward having been shown to be an effective difference (Long and Platt, 2005; Stevens et al., 2005), this was also used in the temporal delay task with dogs (Wright et al., 2012). Reward value has also been examined in non-human animals in terms of having a magnitude effect on rates of discounting and while some evidence suggests there is not a magnitude effect in terms of absolute values (Green et al., 2004; Stevens et al., 2005), there is evidence to support the idea that less preferred rewards are discounted more steeply in rats (Farrar et al., 2003). As different reward values or types may alter the maximum delay an individual tolerates, this could potentially be applied to lengthen or shorten the spatial discounting 
behaviour test in practice, perhaps providing an even more concise way to assess impulsivity in the field. Whether reward value changes the delay tolerated compared to ratio could provide information on the behavioural expression of impulsivity, such as whether the behavioural expression of the trait in dogs (in terms of delay tolerance) can be manipulated by external reinforcers, as suggested in certain situations for rats (Farrar et al., 2003).

Fast and simple assessment enables the spatial discounting task to be used more widely. This makes it a more practical option for the selection of dogs and developing an understanding of the trait in terms of working dog success. As high impulsivity has been linked to behaviour problems resulting from a lack of self-control or inability to tolerate frustration (Wright et al., 2011), it could also have use in the identification of behaviour problems that may present in the field that could be missed during conventional selection and training processes. Dogs might also be a useful model species for the assessment of the influence of self-control training on trait impulsivity in real world settings, given their cohabitation with owners.

It is important to also recognise the applications for this research in other areas where there may not be consistent caregivers for a particular dog, such as a shelter environment. Therefore, in this sort of environment, the Simplified Field Study could be used to provide a useful insight into impulsivity, and the corresponding behavioural prevalence likely associated with certain levels of the trait (Wright et al., 2011). By doing this spatial discounting task on dogs whilst they are in the shelter, then retesting and using a questionnaire once they are in a stable home, it could reveal whether this is a reliable tool for aiding in behaviour assessments. However, it may also be that for some dogs, the environment of the kennels impacts on the results of the behaviour test which may then impact upon the reliability. Therefore, further study is suggested in this area.

Previous tests, such as the temporal discounting task, are more limited in their application as training history impacted on ability to learn the task and so resulted in a potential bias in the form of a selfselecting population (Wright et al., 2012). By contrast the spatial discounting task required little training of the dogs, with at least $96 \%$ meeting the preselection criterion.

However, there are still challenges to assessing impulsivity in young dogs, the age at which the trait becomes stable and measurable remains unknown. No significant relationship was found between young dog MDT and OQS. The OQS was stable over time (4-6 weeks later), while the MDT was not. This suggests that the age at which the behaviour test can be used to predict the OQS score remains unknown. However, as the behaviour test appears to be able to predict impulsivity score in adult dogs, then logically there will be a point at which the test becomes predictive, and there are potentially other factors influencing the behaviour test in young dogs. This could be because owners' opinions become fixed early on rather than the trait becoming stable, or it could be that young dogs are physiologically less able to perform the task due to satiety or fatigue. If impulsivity is not as fixed in young dogs as it is in adult dogs, this would indicate there is potential to shape the expression of the trait towards an optimal phenotype for the animal's future working function. Due to the varied ages of the young dogs sampled in this study there is insufficient data to specifically investigate age effect, but this is worthy of future study. More research is also needed to establish if a test can be devised that can predict overall questionnaire score for impulsivity in young dogs as well as determine at what age the spatial discounting task becomes predictive of this.

\section{Conclusions}

The spatial discounting task has been shown to be a valid measure of OQS for impulsivity based on the DIAS in adult dogs. It is a preferable test to use when compared to previous methods of assessing the trait impulsivity (Wright et al., 2012) as the spatial discounting task takes less time to train and test the dogs and results in a higher completion of test rate. The simplified field version of the spatial discounting task can be used to assess impulsivity in dogs where there are practical limitations, this enables the tool to be more widely used in a field setting. The test also enables in the assessment of impulsivity in dogs where the DIAS cannot be completed. While the OQS was found to be a reliable tool for measuring impulsivity in young dogs of known history, more research is needed to establish the age at which the spatial discounting task shows convergent validity with OQS score for impulsivity.

\section{Acknowledgements and funding}

Naomi Mitchell and Laura Adkins for repeatedly assisting with handling dogs during data collection.

Funding was provided by the UK Ministry of Defence.

\section{References}

Ainslie, G., 1974. Impulse control in pigeons. J. Exp. Anim. Behav. 21, 485-489.

Barratt, E., Patton, J., 1983. Impulsivity: cognitive, behavioural, and psychophysiological correlates. In: Zuckerman, M. (Ed.), Biological Basis of Sensation-Seeking, Impulsivity, and Anxiety. Lawrence Erlbaum Associates, Hillsdale: NJ, pp. 77-116.

Bickel, W., Miller, M., Yi, R., Kowal, B., Lindquist, D., Pitcockre, J., 2007. Behavioural and neuroeconomics of drug addiction: competing neural systems and temporal discounting processes. Drug Alcohol Depend. 90 (1), 85-91.

Brady, K., Myrick, H., McElroy, S., 1998. The relationship between substance use disorders, impulse control disorders, and pathological aggression. Am. J. Addict. 7 (1), 221-230.

Critchfield, T., Kollins, S., 2001. Temporal discounting: basic research and the analysis of socially important behaviour. J. Appl. Behav. Anal. 34 (1), 101-122.

Dalley, A., Marr, A., Economidou, D., Robbins, T., 2008. Neurobehavioural mechanisms of impulsivity: fronto-striatal systems and functional neurochemistry. Pharmacol. Biochem. Behav. 90 (1), 250-260.

De Wit, H., 2008. Impulsivity as a determinant and consequence of drug use: a review of underlying processes. Addict. Biol. 14 (1), 22-31.

Dittmar, H., Bond, R., 2010. I want it and I want it now': Using a temporal discounting paradigm to examine predictors of consumer impulsivity. Br. J. Psychol. 101 (1), $751-776$.

Evans, T., Beran, M., 2007. Chimpanzees use self-distraction to cope with impulsivity. Biol. Lett. 3, 599-602.

Fairbanks, L., Newman, T., Bailey, J., Jorgensen, M., Breidenthal, S., Ophoff, R., Comuzzie, A., Martin, L., Rogers, J., 2004. Genetic contributions to social impulsivity and aggressiveness in vervet monkeys. Biol. Psychiatry 55 (1), 642-647.

Farrar, A., Kieres, A., Hausknecht, K., de Wit, H., Richards, J., 2003. Effects of reinforcer magnitude on an animals model of impulsive behaviour. Behav. Process. 64 (1), $261-271$.

Green, L., Myerson, J., Holt, D., Slevin, J., Estle, S., 2004. Discounting of delayed food rewards in pigeons and rats: is there a magnitude effect? J. Exp. Anal. Behav. 81 (1), 39-50.

Heath-Lang, S., Ha, J., Sackett, G., 1999. Behavioural measurement of temperament in male nursery-Raised infant macaques and baboons. Am. J. Primatol. 47 (1), 43-50.

Kralik, J., Sampson, W., 2012. A fruit in the hand is worth many more in the bush: steep spatial discounting by free-ranging rhesus macaques (Macaca mulatta). Behav. Process. 89 (1), 197-202.

Logue, A., Chavarro, A., Rachlin, H., Reeder, R., 1988. Impulsiveness in pigeons living in the experimental chamber. Anim. Learn. Behav. 16 (1), 31-39.

Long, A., Platt, M., 2005. Decision making: the virtue of patience in primates. Curr. Biol. 15 (21), 874-876.

Moeller, G., Barratt, E., Dougherty, D., Schmitz, J., Swann, A., 2001. Psychiatric aspects of impulsivity. Am. J. Psychiatry 158 (11), 1783-1793.

Muhlhoff, N., Stevens, J., Reader, S., 2011. Spatial discounting of food and social rewards in guppies (Poecilia reticulate). Front. Psychol. 2 (68), 1-11.

Ostaszewski, P., 1996. The relation between temperament and discounting. Eur. J. Pers. 10 (1), 161-172.

Papale, A., Stott, J., Powell, N., Regier, P., Redish, D., 2012. Interactions between deliberation and delay discounting in rats. Cogn. Affect. Behav. Neurosci. 12 (1), 513-526.

Pavlov, K., Chistiakov, D., Chekhonin, V., 2012. Genetic determinants of aggression and impulsivity in humans. J. Appl. Genet. 53 (1), 61-82.

Peremans, K., Audentart, K., Coopman, F., Hoybergs, Y., Slegers, G., Van Bree, H., Verschooten, F., Dierckx, R., 2002. Functional brain imaging of serotonin-2a receptors in impulsive dogs: a pilot study. Flemish Vet. J. 71 (1), 340-347.

Perry, J., Larson, E., German, J., Madden, G., Carroll, M., 2005. Impulsivity (delay discounting) as a predictor of acquisition of IV cocaine self-administration in female rats. Psychopharmacology 178 (1), 193-201.

Perry, J., Nelson, S., Anderson, M., Morgand, A., Carrol, M., 2007. Impulsivity (delay discounting) for food and cocaine in male and female rats selectively bred for high and low saccharin intake. Pharmacol. Biochem. Behav. 86 (1), 822-837.

Pine, A., Shiner, T., Seymour, B., Dolan, R., 2010. Dopamine, time and impulsivity in humans. J. Neurosci. 30 (26), 8888-8896.

Putnam, S., Ellis, L., Rothbart, M., 2001. The structure of temperament from infancy 
through adolescence. In: Aliasz, A., Angleitner, A. (Eds.), Advances in Research on Temperament. Pabst Science, Germany, pp. 165-182.

Puumala, T., Sirvo, J., 1999. Changes in activities of dopamine and serotonin systems in the frontal cortex underlie poor chance accuracy and impulsivity if rats in an attention task. Neuroscience 83 (2), 489-499.

Reimer, S., Mills, S., Wright, H., 2014. Impulsive for life? The nature of long -term impulsivity in domestic dogs. Anim. Cogn. 17, 815-819.

Reynolds, B., Wit, H., Richards, J., 2002. Delay of gratification and delay discounting in rats. Behav. Process. 59 (1), 157-168.

Rosati, A., Stevens, J., Hare, B., Hauser, M., 2007. The evolutionary origins of human patience: temporal preferences in chimpanzees, bonobos, and human adults. Curr. Biol. 17 (19), 1663-1668.

Stevens, J.R., Rosati, A.G., Ross, K.R., Hauser, M.D., 2005. Will travel for food: spatial discounting in two new world monkeys. Curr. Biol. 15 (20), 1855-1860.

Svobodová, I., Vápeník, P., Pinc, L., Bartoš, L., 2008. Testing German shepherd puppies to assess their chances of certification. Appl. Anim. Behav. Sci. 113 (1-3), 139-149.
Tobin, H., Logue, A., Chelonis, J., Ackerman, K., 1996. Self-control in the monkey Macaca fascicularis. Anim. Learn. Behav. 24, 168-174.

Vollmer, S., Borrero, J., Lalli, J., Daniel, D., 1999. Evaluating self-control and impulsivity in children with severe behavioural disorders. J. Appl. Behav. Anal. 32 (1), 451-466. Wilsson, E., Sundgren, P., 1998. Behaviour test for eight-week old puppies—heritabilities of tested behaviour traits and its correspondence to later behaviour. Appl. Anim. Behav. Sci. 58 (1-2), 151-162.

Wolff, M., Leander, D., 2002. Selective serotonin reuptake inhibitors decrease impulsive behaviour as measured by an adjusting delay procedure in the pigeon. Neuropsychopharmacology 27 (3), 421-429.

Wright, H., Mills, D., Pollux, P., 2011. Development and validation of a psychometric tool for assessing impulsivity in the domestic dog (Canis familiaris). Int. J. Comp. Psychol 24 (1), 210-225.

Wright, H., Mills, D., Pollux, P., 2012. Behavioural and physiological correlates of impulsivity in the domestic dog (Canis familiaris). Physiol. Behav. 105 (3), 676-682. 